\title{
An RNA interference screen identifies new avenues for nephroprotection
}

\author{
ER Zynda ${ }^{1}$, B Schott ${ }^{2}$, S Gruener ${ }^{2}$, E Wernher ${ }^{2}$, GD Nguyen ${ }^{3}$, M Ebeling $^{3}$ and ES Kandel ${ }^{*, 1}$
}

Acute kidney injury is a major public health problem, which is commonly caused by renal ischemia and is associated with a high risk of mortality and long-term disability. Efforts to develop a treatment for this condition have met with very limited success. We used an RNA interference screen to identify genes (BCL2L14, BLOC1S2, C2ORF42, CPT1A, FBP1, GCNT3, RHOB, SCIN, TACR1, and TNFAIP6) whose suppression improves survival of kidney epithelial cells in in vitro models of oxygen and glucose deprivation. Some of the genes also modulate the toxicity of cisplatin, an anticancer agent whose use is currently limited by nephrotoxicity. Furthermore, pharmacological inhibition of TACR1 product NK1R was protective in a model of mouse renal ischemia, attesting to the in vivo relevance of our findings. These data shed new light on the mechanisms of stress response in mammalian cells, and open new avenues to reduce the morbidity and mortality associated with renal injury.

Cell Death and Differentiation (2016) 23, 608-615; doi:10.1038/cdd.2015.128; published online 13 November 2015

Acute kidney injury (AKI), also known as acute renal failure, is an abrupt decrease in kidney function characterized by accumulation of creatinine and urea in the blood. It is a global clinical problem with increasing incidence, dire consequences, unsatisfactory therapeutic options, and an enormous financial burden to societies worldwide. ${ }^{1,2}$ The reported prevalence of $\mathrm{AKI}$ varies from $1 \%$ to upward of $25 \%$ depending on the diagnostic criteria and the study population, with especially high incidence among the patients of intensive care units. ${ }^{2,3}$ The mortality of these patients may approach or even exceed $50 \%$, and those that survive face prolonged hospitalization and significant increases in morbidity. ${ }^{3}$

Renal ischemia is the major cause of $\mathrm{AKI}^{4}$ and typically develops following a drop in blood flow to the kidney, causing hypoxia and nutrient deprivation within the affected organ. Although most organs compensate for a reduction in oxygenation by increasing local blood flow, the hypoxic kidney does not increase perfusion, ${ }^{5}$ likely because blood does not simply provide sustenance, but also serves as a substrate for energy-consuming renal filtration. Instead, hypoxic kidneys influence blood oxygen content by stimulating erythropoiesis via release of erythropoietin. ${ }^{6}$ However, this process is relatively slow, making metabolically active kidney cells highly vulnerable to ischemia.

The initial damage from ischemia is further amplified during reperfusion. The ensuing inflammation creates an environment favorable for fibrosis, which, in turn, permanently attenuates renal function. ${ }^{7}$ Chronic kidney disease greatly increases the incidence of new episodes of $\mathrm{AKI}$, thus completing the vicious cycle of organ destruction. ${ }^{8}$ Patients undergoing cardiac surgery are known to be at a high risk for ischemic AKI, ${ }^{9}$ as are the individuals suffering from sepsis, ${ }^{10}$ dehydration ${ }^{11}$ and many other conditions. Kidney ischemia is also a prominent factor for kidney transplant patients. In fact, the duration of ischemia experienced by the transplanted kidney is a major predictor for transplant success or failure. ${ }^{12}$

The enormous public health significance has attracted considerable effort to the development of strategies to prevent or reduce the damage from ischemic AKI. ${ }^{7,13}$ Current treatment options are aimed at controlling known risk factors in highly susceptible populations or at attempting to replace renal function in patients who have lost it due to injury. ${ }^{14}$ Despite major advances in our understanding of the pathology of ischemic injury, current therapies are merely supportive. To the best of our knowledge, no available therapy has been definitively proven to effectively avert the damage to an ischemic kidney. ${ }^{4}$ This critical deficiency in the arsenal of modern medicine prompted us to seek suitable pharmacologic targets, which could be exploited for such nephroprotection.

We report here the identification of 10 genes, interference with which protects immortalized renal proximal epithelial cells in an in vitro model of ischemia. We also report that some of

${ }^{1}$ Department of Cell Stress Biology, Roswell Park Cancer Institute, Elm and Carlton Streets, Buffalo, NY 14263, USA; ${ }^{2}$ Department of Cardiovascular and Metabolic Discovery, F. Hoffmann-La Roche Ltd, Bau 70/307, Basel CH4070, Switzerland and ${ }^{3}$ Department of Pharmaceutical Sciences, Translational Technologies and Bioinformatics, F. Hoffmann-La Roche Ltd, Bau 70/307, Basel CH4070, Switzerland

${ }^{*}$ Corresponding author: ES Kandel, Department of Cell Stress Biology, Roswell Park Cancer Institute, Elm and Carlton Steets, Buffalo, NY 14221, USA. Tel: 716845 3530; Fax: 716845 3944; E-mail: Eugene.Kandel@RoswellPark.org

Abbreviations: AKI, acute kidney injury; shRNA, short hairpin ribonucleic acid; BCL2L14, B-cell lymphoma 2-like 14; BLOC1S2, Biogenesis of lysosomal organelles complex-1, subunit 2; C2ORF42, Chromosome 2, open-reading frame 42; CPT1A, Carnitine palmitoyltransferase 1A; FBP1, Fructose-1,6-bisphosphatase 1; GCNT3, Glucosaminyl (N-acetyl) transferase 3; RHOB, Ras homolog family member B; SCIN, Scinderin; TACR1, Tachykinin receptor 1; TNFAIP6, Tumor necrosis factor, alphainduced protein 6; NK1R, neurokinin 1 receptor; TAC1, Tachykinin, precursor 1; HIF-1, hypoxia-inducible factor-1; TRAIL, TNF-related apoptosis-inducing ligand; MAPK, Mitogen-activated protein kinases; CD44, cluster of differentiation 44; GTP, guanosine-5'triphosphate; BCL-G, B-cell lymphoma-G; FAU, Finkel-Biskis-Reilly murine sarcoma virus ubiquitously expressed; PAXIP1, Paired box 1 interacting (with transcription-activation domain) protein 1; ECT2, Epithelial cell transforming 2; RIST1, Regulator of ischemia and stress tolerance 1; RT-PCR, Reverse transcription PCR

Received 18.5.2015; revised 03.8.2015; accepted 20.8.2015; Edited by G Melino; published online 13.11.15 
the genes may be involved in the response to other cytotoxic stresses, and that chemical inhibition of the product of one of the genes reduces the extent of ischemic AKI in animals.

\section{Results}

Identification of shRNAs that protect kidney epithelial cells in a model of ischemia. Proximal tubule cells are particularly susceptible to ischemia. ${ }^{15} \mathrm{~A}$ very high metabolic rate is required for mediating ion transport, and these cells have a severely limited capacity for anaerobic glycolysis. ${ }^{15}$ Importantly, these cells tend to accumulate toxic by-products of incomplete fatty acid oxygenation, and remain hypoxic for an extended period of time, well after reperfusion. ${ }^{16}$ Our effort was inspired by the observations that the death of hypoxic kidney cells is an active process, both in the sense of metabolic maladaptations that inflict the initial biochemical insult, and in the sense of mechanisms that recognize the damage and commit cellular pathways to cell death. ${ }^{7,13,17}$ This suggests a possibility of nephroprotection via inhibition of certain cellular factors. RNA interference-based genetic approaches are well suited for discovering such factors. ${ }^{18} \mathrm{We}$ used the non-transformed proximal tubule epithelial cell line, $\mathrm{HK}-2,{ }^{19}$ in conditions of low oxygen and glucose as an in vitro model of AKI. HK-2 cells are commonly used to study the mechanisms of cell death in renal epithelium. ${ }^{20}$

We screened a pooled lentiviral library containing $\sim 80000$ shRNAs targeting $\sim 16000$ human genes for enhanced survival under low-oxygen, low-glucose conditions (designated as 'ischemic' for the purpose of this study), and chose 23 genes for further examination (Supplementary Figure 1; Supplementary Table 1). The tests confirmed that individual interference with 10 of these genes confers robust protection in our in vitro model (Figures 1a and d). More than a fivefold increase in the number of remaining cells was achieved by shRNAs that were not a part of the originally screened pool, confirming that the phenomenon reflects the properties of the intended target genes, and not off-target effects of individual shRNAs. The list of genes included: BCL2L14, BLOC1S2, C2ORF42, CPT1A, FBP1, GCNT3, RHOB, SCIN, TACR1, and TNFAIP6. We have tested the efficacy of target suppression by several of the protective shRNAs, and have observed a significant reduction in the levels of expression of the respective genes (Supplementary Figure 2). Importantly, using $R H O B$-targeting shRNAs as an example, we documented that the more efficient shRNAs also render better protection (Figure 1b). In order to exclude cell line specificity of our findings, we repeated the assays using another human a

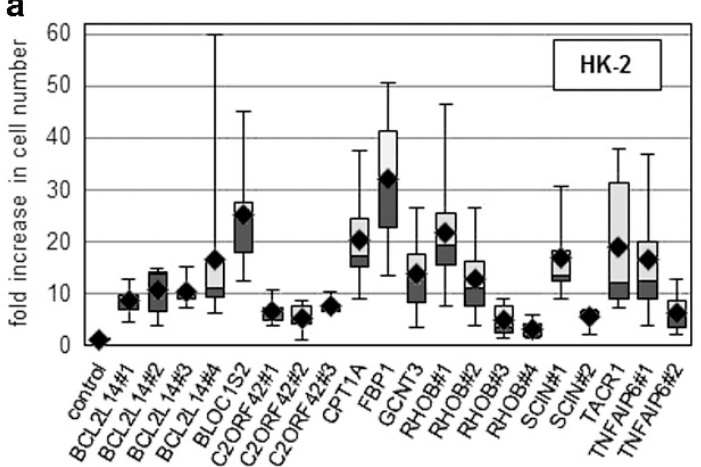

b

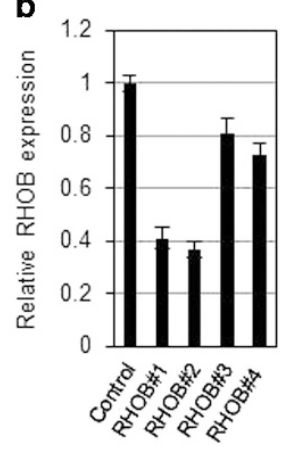

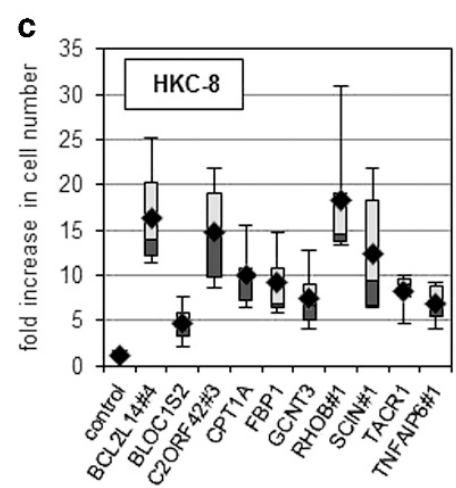

d

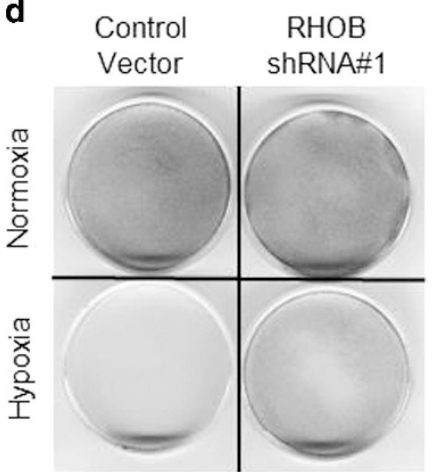

Figure 1 Candidate shRNAs confer resistance to hypoxia in HK-2 cells. (a) HK-2 cells expressing candidate shRNAs were subjected to oxygen and glucose deprivation for $48 \mathrm{~h}$. Following treatment, cell numbers for each culture were assessed relative to respective normoxic controls, and values are presented relative to those in parental cells. Cumulative results from at least six independent experiments are shown for each construct. Black rhombuses depict geometric means. Lightly and darkly shaded boxes depict second and third percentiles, respectively. Error bars indicate the ranges. The differences between every individual shRNA and the control vector are significant $(P<0.05)$. (b) $R H O B$ mRNA levels in HK-2 cells transduced with four different $R H O B$-specific shRNAs were measured by qRT-PCR, normalized to mean GAPDH transcript levels and reported relative to that in cells transduced with the control vector (pGIPZ). The means and S.D. of three independent experiments are shown. (c) HKC-8 cells expressing candidate shRNAs were subjected to oxygen and glucose deprivation for $48 \mathrm{~h}$. The data for three separate experiments were processed and presented as in a. The differences between every individual shRNA and the control vector are significant $(P<0.05)$. (d) Representative images of parallel cultures of HK-2 cells, which were transduced with the control vector or a protective shRNA (RHOB\#1). Hypoxia treatment was performed as described in the Materials and methods section, and the remaining cells were visualized by methylene blue staining 
renal proximal tubular cell line, HKC- $8,{ }^{21}$ with the shRNAs that showed efficacy in HK-2 cells. All 10 tested shRNAs showed robust protection under the selective conditions (Figure 1c).

The role of the identified genes in kidney cell response to cisplatin. In addition to ischemia, AKI can result from exposure to toxins, including various therapeutic agents. For example, renal toxicity of cisplatin limits dose escalation for this anticancer compound, prevents the use of this drug in patients with compromised renal function, and may lead to chronic kidney disease in the treated population. ${ }^{22}$ We investigated whether any of the identified genes may affect the response of kidney epithelial cells to cisplatin. Interference with BCL2L14, RHOB and C2ORF42 provided robust protection in HK-2 and HKC-8 cells (Figures $2 a$ and b), whereas interference with TNFAIP6 yields very strong protection in HKC-8 cells only (Figure $2 b$ ), indicating that the mechanisms of drug response are not identical between the cell lines. This is also evident from the difference in cisplatin tolerance between the two parental cell lines (Figures 2a and b).

Although normal kidney epithelium is highly sensitive to cisplatin, clear-cell renal cell carcinoma is notoriously unresponsive to conventional chemotherapy. Interestingly, this remarkable change from drug sensitivity to resistance during tumor evolution is paralleled by the loss of C2ORF42 expression (Figures $2 \mathrm{c}$ and d). ${ }^{23,24}$

Protective effects of NK1R inhibition in vitro and in vivo. Among the identified genes, the product of TACR1 (neurokinin 1 receptor, NK1R) is inhibited by a number of well-characterized drugs, some of which are used clinically. We hypothesized that those compounds would recapitulate the effects of TACR1 shRNA on ischemia tolerance. Indeed, NK1R antagonists, L-733,060 and Aprepitant, conferred resistance to ischemia in both HK-2 and HKC-8 cells (Figures $3 a$ and $d$ ) at concentrations that had minimal effect on the survival of normoxic cells (Supplementary Figures 3a and d). Furthermore, significant protection was achieved by knockdown of TAC1, which encodes for a known NK1R ligand, substance $\mathrm{P}$ (Supplementary Figure 4a).

We further evaluated the consequences of NK1R inhibition in an in vivo model of acute renal ischemia. Mice pretreated with $30 \mathrm{mg} / \mathrm{kg}$ or $90 \mathrm{mg} / \mathrm{kg}$ of L-733,060 or the respective vehicle were subjected to transient ischemia on both kidneys. After $24 \mathrm{~h}$ of reperfusion, measurement of blood-based markers of AKI revealed a dose-dependent protection by NK1R inhibition (Figure 4). Levels of creatinine and blood urea nitrogen were significantly $(P<0.05$, Mann-Whitney test) reduced by either dose of the compound (Figures $4 a$ and $b$ ), whereas the levels of neutrophil gelatinase-associated lipocalin and osteopontin were significantly reduced by a higher dose $(P<0.05)$. The same trend appeared at the lower dose, albeit without reaching statistical significance (Figures $4 c$ and d).

Interference with $R H O B$ and C2ORF42 increases the anti-apoptotic effect of Aprepitant. It is clear that multiple processes contribute to cell death during AKI, and simultaneous targeting of multiple pathways may be needed for maximal protection. ${ }^{13}$ To this end, we investigated whether interference with any of the confirmed mediators of ischemic death could further improve the peak protection, which is rendered by chemical inhibition of NK1R. Indeed, we observed that interference with C2ORF42 or RHOB consistently increased the maximal efficacy of the drugs (Figures $5 \mathrm{a}$ and b; Supplementary Figure 5).

Apoptosis is reported to be the predominant mode of cell death in response to ischemia. ${ }^{15}$ Accordingly, we observed that the degree of protection from ischemia correlates well with a reduction in caspase activity in these treatment groups (Figure 5c).

\section{Discussion}

A decade ago it was argued that an effective approach to limit or prevent ischemic renal injury in humans remains elusive, primarily because of an incomplete understanding of the mechanisms of cellular injury. ${ }^{25}$ Although molecular nephrology continues to progress, mechanisms that may protect ischemic tubular epithelial cells remain poorly understood. The matter is complicated by the fact that the known cell programs, which improve survival in hypoxia (e.g., the hypoxia-inducible factor-1 (HIF-1)-mediated switch from oxidative phosphorylation toward glycolysis) or hypoglycemia (e.g., the switch from glucose to lipid oxidation), become maladaptive when these conditions coincide. Indeed, HIF-1 may also become pro-apoptotic, ${ }^{26,27}$ and the toxic products of partial lipid metabolism contribute to ischemic injury. ${ }^{28}$

It is known that reperfusion amplifies the initial injury. This secondary damage ensues from inflammation, and the release of molecules such as osteopontin by injured cells may serve to attract the immune system. ${ }^{29}$ Subsequent fibrosis may preserve overall organ integrity, but diminish its function. Again, HIF-1 activity may be maladaptive at this stage, contributing to inflammation, tubular atrophy, and fibrosis. ${ }^{30}$ Importantly, it is likely that avoiding the initial damage to epithelial cells would also prevent damage following reperfusion. An additional reason for targeting primary rather than secondary damage in renal ischemia is that mechanisms of ischemic cell death appear conserved, whereas the particulars of reperfusion injury and the modes of its aversion differ between species ${ }^{16}$ and even within species, ${ }^{31}$ which limits inferences from animal models.

The recognition of large cohorts of individuals at risk for AKI stimulates the push for treatments that would prevent the injury, rather than mitigate its consequences. Multiple promising strategies, too numerous to be listed here, are aimed at averting AKI by rational metabolic or immune interventions, but have proven to be ineffective or unacceptably toxic in preclinical models and clinical trials. ${ }^{13,32}$ Undoubtedly, many new approaches are still at various stages of pre-clinical development, but we are unaware of any of them that have successfully crossed the threshold of clinical testing. Similarly, a large number of targeted strategies intended to mitigate the consequence of ischemia in transplant patients have yielded controversial or negative results. ${ }^{30}$

Of note, the earlier attempts at nephroprotection focused on a handful of molecules and pathways, which have been extensively characterized in prior research. In contrast, we undertook an unbiased approach, as we screened a 

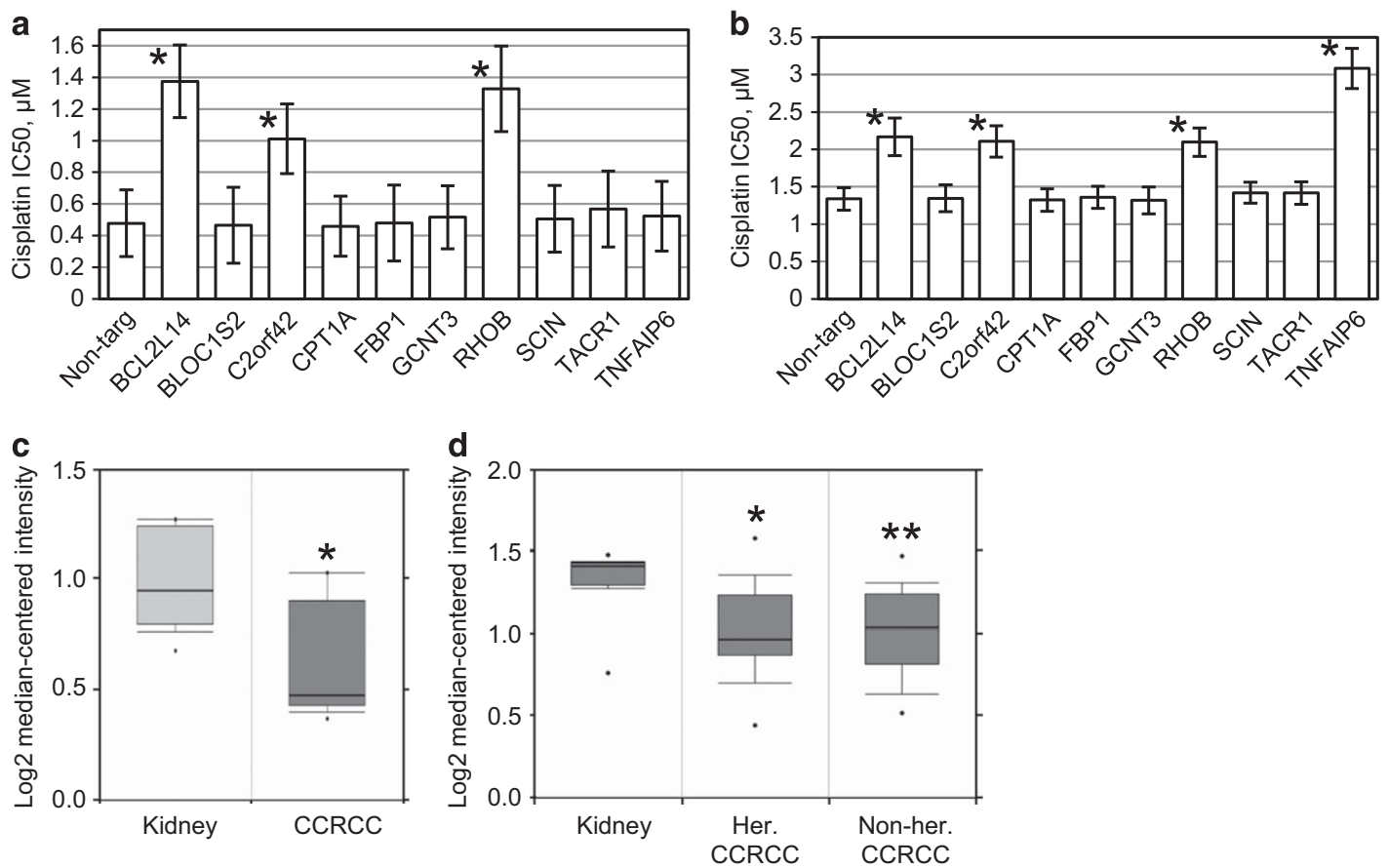

Figure 2 Effects of candidate shRNAs expression on cisplatin-induced cytotoxicity. HK-2 cells (a) and HKC-8 cells (b), which harbored a control non-targeting shRNA or the shRNAs against the indicated genes, were treated for $48 \mathrm{~h}$ with cisplatin concentrations ranging from 0 to $25.6 \mu \mathrm{M}$, followed by 3 days in drug-free medium. For each culture, numbers of remaining cells were compared with those in respective untreated controls using methylene blue staining and the extraction method. The bars represent the $\mathrm{IC}_{50}$ values. The error bars depict 95\% confidence intervals. Asterisks indicate a significant $(P<0.05)$ increase in $\mathrm{IC}_{50}$ over the respective controls. (c) C2ORF42 expression is commonly decreased in clear-cell renal cell carcinoma (CCRCC). The data were retrieved from www.oncomine.com., ${ }^{*} P=0.001$. (d) $\mathrm{C} 2 \mathrm{ORF} 42$ is decreased in both hereditary (her.) and non-hereditary (non-her.) CCRCC. ${ }^{*} P=1.41 \mathrm{E}-4,{ }^{* *} P=2.28 \mathrm{E}-4$. The data were retrieved from www.oncomine.com

genome-wide shRNA library. Screening such a complex library is vulnerable to stochastic experimental 'noise' and various well-recognized artifacts of shRNA technology. ${ }^{18,33}$ Thus, it is imperative to validate any candidate genes by additional tests using either individual shRNAs or alternative approaches, for example, chemical inhibitors. We have validated the effect for 10 out of 23 candidate genes. We cannot conclude that the 13 non-validated genes are irrelevant to ischemic response: the negative results could have ensued from insufficient efficacy of the particular shRNAs, or from adverse off-target effects of these shRNAs that outweighed the protective action. Also, our stringent threshold (fivefold increase in cell number over unprotected control) might have excluded relevant genes with weaker effects. In addition, many relevant genes may have been missed during the initial screening, for example, due to insufficient potency or off-target toxicity of the corresponding shRNAs. Furthermore, the discovery of genes, whose products may be nephroprotective upon upregulation, would require an entirely different set of tools. Nevertheless, the discovery of the 10 modulators of nephrotoxicity provides a starting point for elucidating the relevant pathways using conventional biochemical and genetic approaches.

The identified modulators of nephrotoxicity differ in the biochemical properties of their products and in how well they have been characterized. To further complicate matters, information about their function comes from different cell systems, whereas the specifics of hypoxic cell death may differ even between closely related cell types. ${ }^{34}$
Among these genes, TACR1 is notable due to the availability of clinically useful inhibitors of its product. Although much more work is needed to confirm clinical utility of these agents against $\mathrm{AKI}$, our in vivo observations are encouraging. These results also indicate that our in vitro system is capable of producing information relevant to renal ischemia in a wholeorganism context. Inhibition of NK1R has been proposed as an avenue of ischemia protection for brain and heart, ${ }^{35,36}$ but was viewed as a way of preventing secondary damage from edema and other complications of reperfusion. In contrast, our in vitro findings demonstrate that this intervention, at least in the context of kidney epithelial cells, prevents primary damage in the absence of additional cell types. Accordingly, a combination of NK1R and its ligand was reported to kill human neurons and embryonic kidney cells. ${ }^{37}$ Unlike our system (Figure 5c), the reported mode of death was non-apoptotic. ${ }^{37}$ However, the choice between apoptotic and non-apoptotic programs may be decided by the availability of various cellular factors after the cell has committed to dying. ${ }^{38}$

Although the precise nature of the death-promoting ligand has yet to be elucidated, the fact that $\mathrm{HK}-2$ cells are protected by an shRNA targeting TAC1 (Supplementary Figure 4a) suggests that a product of that gene (e.g., substance $P$ ) might be involved in triggering cell death. A relatively small protective effect of TAC1 knockdown could be explained by insufficient potency of the shRNA, by the presence of cells in which the shRNA expression construct is aberrant or poorly expressed (such cells would still secrete substance $\mathrm{P}$ and affect their neighbors), or by the existence of other pro-apoptotic NK1R 
ligands. Interestingly, the naked mole rat, which is adapted to hypoxic environments, has extremely low levels of substance $P^{39}$

Intriguingly, we observed that interference with TACR1 affects GCNT3 expression (Supplementary Figure 4b). As GCNT3 inhibition is also protective, it is possible that TACR1dependent induction of GCNT3 contributes to ischemic cell
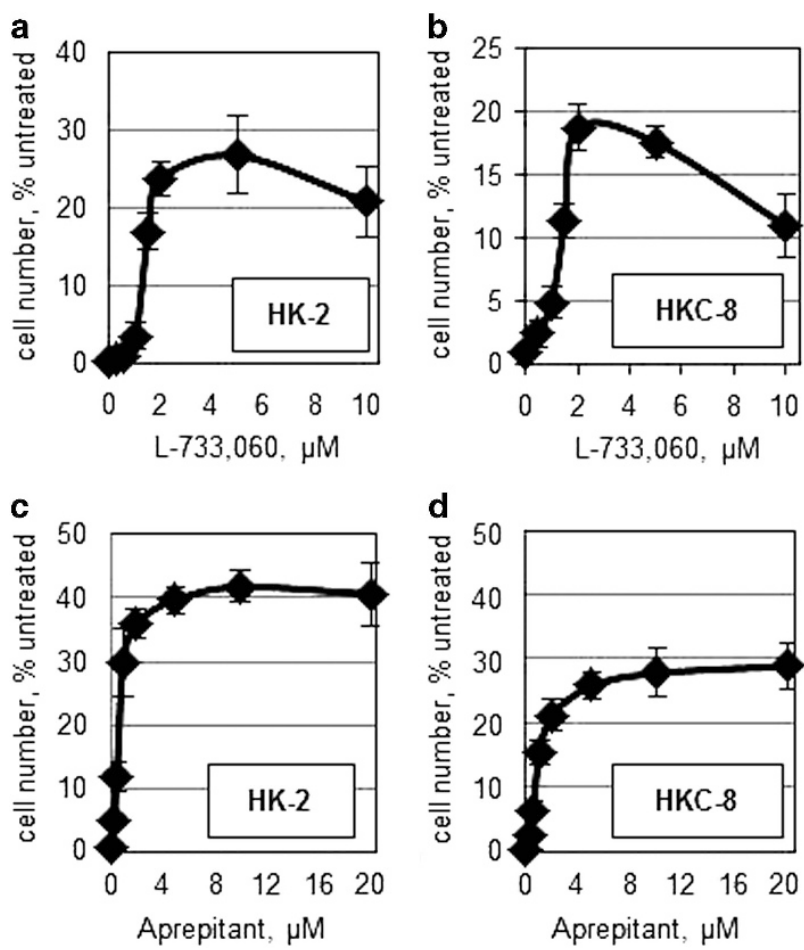

Figure 3 The effects of the NK1R antagonists on hypoxia resistance in kidney epithelial cells. (a and b) HK-2 (a) and HKC-8 (b) were treated with L-733,060 and exposed to ischemic conditions for $48 \mathrm{~h}$. For each dose of the drug, the number of cells remaining after treatment was graphed as a percent of cells kept under normoxic conditions. The experiemnts were conducted in triplicate. The error bars depict S.D. (c and d) HK-2 cells (c) and HKC-8 cells (d) were treated with Aprepitant and exposed to ischemic conditions for $48 \mathrm{~h}$. The data were processed and presented as in $\mathbf{a}$ and $\mathbf{b}$. In both cell lines, significant $(P<0.05)$ protection was observed at concentrations of $1 \mu \mathrm{M}$ and above for L-733,060 and $0.25 \mu \mathrm{M}$ and above for Aprepitant
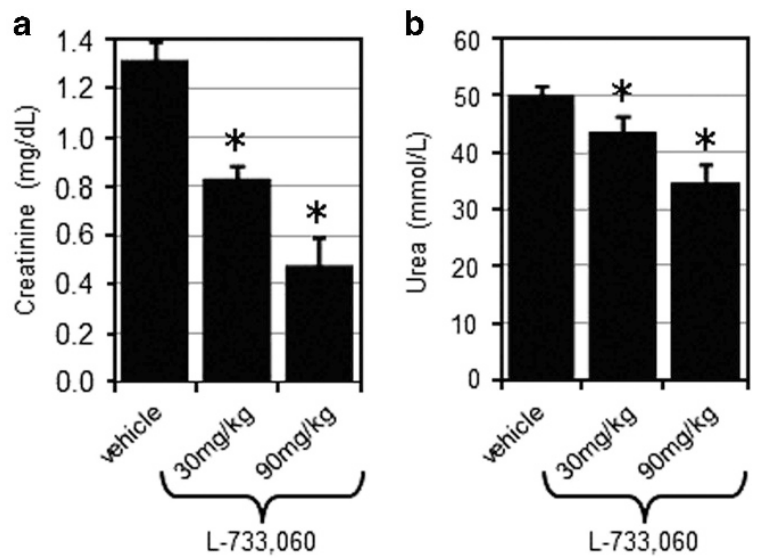

death. Importantly, GCNT3-deficient mice are viable and fertile, despite some defects in immune functions. ${ }^{40}$ Thus, transient inhibition of GCNT3 is likely to be well tolerated.

At this time the mechanism of involvement of TACR1 and GCNT3 in ischemic cell death is unknown. TNF-related apoptosis-inducing ligand (TRAIL) expression is derepressed by hypoxia, at least, in some cells, ${ }^{41}$ and there is evidence that cell death in an ischemic kidney depends on TRAIL signaling. ${ }^{42}$ We can also speculate that $\mathrm{N}$-acetylglucosaminyltransferase GCNT3 contributes to the glycosylation of TRAIL receptors, which is critical for their function. ${ }^{43,44}$ In addition, substance $P$ stimulates $p 21$-activated kinase, ${ }^{45}$ which is a known regulator of the mitogen-activated protein kinases (MAPK) cascade. ${ }^{46}$ In turn, MAPKs, at least in some cases, may sensitize cells to TRAIL-induced apoptosis. ${ }^{47} \mathrm{We}$ believe that the hypothetical connection between TACR1 and TRAIL-mediated cell death is worth exploring, because the likely intermediaries include multiple drug-able proteins.

Another gene identified in our study, FBP1, encodes a metabolic enzyme, inhibition of which may lead to more efficient utilization of limited resources. ${ }^{48} \mathrm{~A}$ recently reported role of FBP1 in the control of gene expression ${ }^{49}$ may also have a role in this process.

CPT1A also encodes a metabolic enzyme. Its product contributes to fatty acid oxidation, and its inhibition may be 'decreasing the oxygen cost of adenosine triphosphate production', 50 as well as preventing the accumulation of toxic, partially oxidized products. Manipulation of CPT enzymes has been considered as a potential therapeutic strategy for treating various diseases, but this is complicated by distinct roles of CPT isoforms as well as the questionable specificity of available inhibitors. ${ }^{51}$

The product of the BLOC1S2 gene was first described as a protein with a role in biogenesis of lysosome-related organelles, but later research suggested that it is needed for efficient execution of apoptosis in glioblastoma cells. ${ }^{52}$

SCIN encodes a calcium-dependent actin filament-severing protein known to have a role in exocytosis. ${ }^{53}$ It acts as a pro-apoptotic protein in megakaryocyte leukemia. ${ }^{54}$

TNFAIP6 is inducible by proinflammatory cytokines, such as tumor necrosis factor- $a,{ }^{55}$ which is a known sensitizer to AKI c

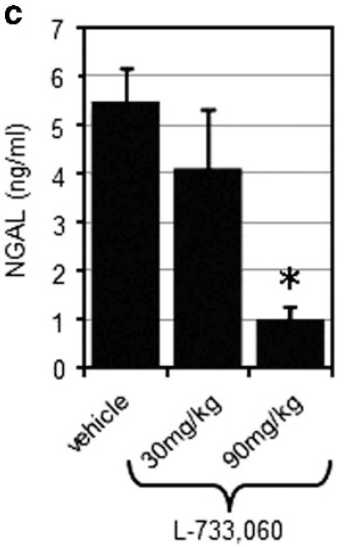

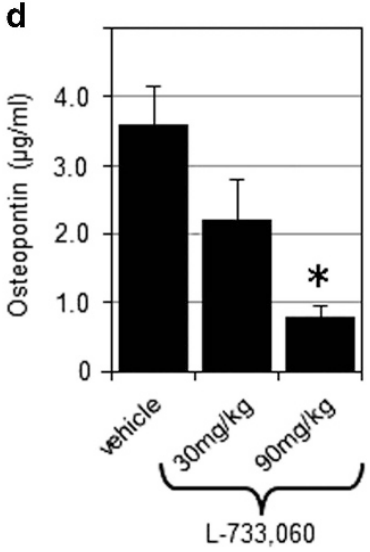

Figure 4 The effects of NK1R inhibitor in a mouse model of kidney ischemia. At 30 min after treatment with the indicated doses of L-733,060, the mice were subjected to $25 \mathrm{~min}$ of bilateral renal ischemia. At $24 \mathrm{~h}$ following reperfusion, serum levels of creatinine (a), urea (b), neutrophil gelatinase-associated lipocalin (NGAL) (c), and osteopontin (d) were measured. The means with S.D. are shown. The asterisks indicate a significant $(P<0.05)$ difference from the vehicle-treated group 
a

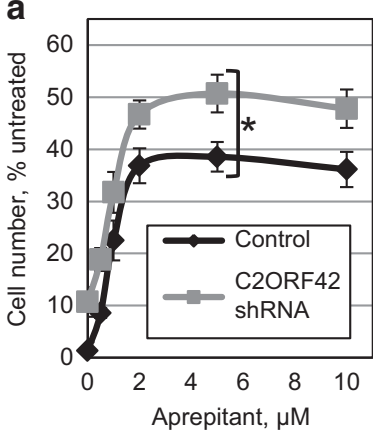

b

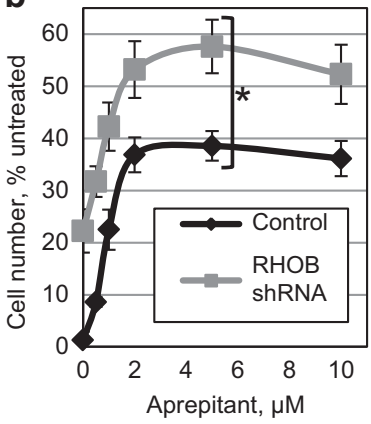

C

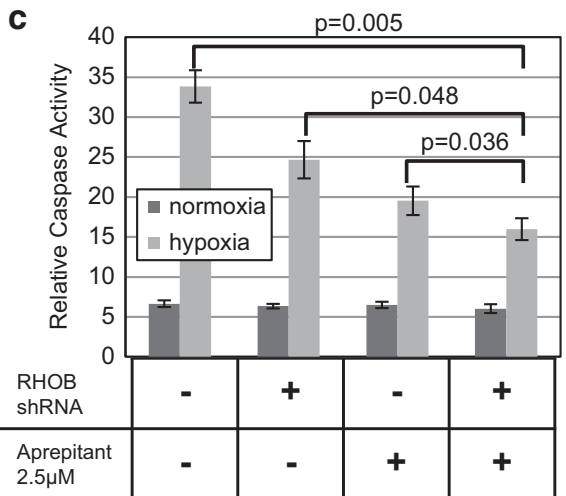

Figure 5 The combination of Aprepitant and select shRNAs enhances hypoxia tolerance in kidney epithelial cells. HK-2 cells expressing shRNA targeting (a) C2ORF42 and (b) RHOB were treated with the indicated concentrations of Aprepitant and exposed to ischemic conditions for $48 \mathrm{~h}$. For each dose of the drug, the numbers of cells remaining after treatment were analyzed as a percent of cells incubated under normoxic conditions. For each shRNA, the results are shown in comparison with those for vector-transduced HK-2 cells, which were treated in parallel. (c) Caspase activity following $40 \mathrm{~h}$ of ischemia was measured in HK-2 cells, modified or treated as indicated. Error bars for all panels indicate S.D. of three independent experiments. The asterisks indicate a significant $(P<0.05$, unless otherwise indicated in panel $\mathbf{c})$ difference comparing the indicated treatment goups

and a possible target for nephroprotection. ${ }^{56}$ Among other roles, TNFAIP6 modulates the interaction between hyaluronan and $\mathrm{CD} 44,{ }^{57}$ both of which are proposed targets for nephroprotection. ${ }^{58,59}$ Importantly, interference with TNFAIP6 reduces epithelial-to-mesenchymal transition in kidney epithelial cells. ${ }^{60}$ In conjunction with our findings, this suggests that anti-TNFAIP6 therapy might prevent both the initial damage during ischemia and post-reperfusion fibrosis.

RhoB is a member of the Rho GTP-binding protein family. Its downregulation has been implicated in resistance of some cancer cells to cisplatin. ${ }^{61}$ RhoB is activated by a variety of stress signals. ${ }^{62}$ In particular, RhoB is activated within minutes of hypoxia in a glioblastoma cell line, ${ }^{63}$ and differential regulation of the $R H O B$ gene was implicated as a mechanism of uncommon tolerance to hypoxia in a subterranean rodent. ${ }^{64}$ Intriguingly, RhoB controls intracellular trafficking, ${ }^{62}$ a process that is reportedly affected by other genes identified in our screen (BCL2L14, SCIN and BLOC1S2), and failure to traffic respective receptors is a mechanism of resistance to deathpromoting cytokines, including TRAIL. ${ }^{65,66}$

B-cell lymphoma-G (Bcl-G), encoded by BCL2L14 gene, has been characterized as a pro-apoptotic Bcl-2 family member. ${ }^{67}$ This model has been challenged recently by the observation that BCL214-deficient dendritic cells are sensitive to apoptosis. ${ }^{68}$ Although this might reflect different roles for this protein in different cells and in response to different stressors, there are clues linking Bcl-G with vesicle transport. ${ }^{68,69}$ Of note, Bcl-G regulator FAU has been under intense selection in hypoxia-adapted mole rats. ${ }^{70}$

C2ORF42 is the least studied gene on our list. It is conserved between mammals and arthropods. Its product reportedly associates with a mediator of the cell stress response, PAXIP1, and a Rho GTPase regulator, epithelial cell transforming 2 (ECT2), ${ }^{71}$ but its biochemical function is unknown. Considering our findings, we propose to name the gene Regulator of Ischemia and Stress Tolerance 1 (RIST1).

In addition to ischemia, exposure to toxins is an important cause of AKI. Nephrotoxicity remains a major side effect of many pharmaceuticals, and patients with chronic kidney disease are especially at risk. Cisplatin is an example of a highly effective drug, for which nephrotoxicity is dose limiting. ${ }^{72}$ The status of at least three of the identified genes ( $R H O B, B C L 2 L 14$, and RIST1) strongly affects the response of renal epithelial cell lines to this drug. RhoB has already been implicated in response to DNA damage in other contexts, ${ }^{61}$ whereas Bcl-G may be a mediator of the drug-sensitizing functions of its regulator, FAU. ${ }^{73}$ Intriguingly, the alleged RIST1-interacting partner, ECT2, also activates RhoB following DNA damage, ${ }^{74}$ which may explain similar outcomes of targeting RIST1 and RHOB in our assays. The hypothesis that RIST1, RHOB and ECT2 belong to the same biological pathway is further supported by the fact that interference with ECT2 has an ischemia-protective effect similar to that of RHOB and RIST1 (Supplementary Figure 6). Overall, our findings encourage inquiries into whether interfering with $R H O B, B C L 2 L 14$, and RIST1 and their partners could protect against cisplatin-induced nephrotoxicity without compromising the anti-tumor effect of this drug, and whether these genes have a wider role in on- and off-target toxicity of various pharmaceuticals.

As resistance to hypoxia is a key step in cancer evolution, the mediators of ischemic cell death reported here may have a role in tumorigenesis and tumor resistance to therapy. For example, the common reduction in RIST1 expression in renal cancer (Figures 2c and d) might offer an explanation for the remarkable resistance of this disease to conventional chemotherapy. Also, the loss of FBP1 has been recently established as an oncogenic event in renal cancer. ${ }^{49}$ However, a possible involvement of these genes in cancer is not necessarily incompatible with the development of strategies for nephroprotection based on their inhibition. First, any protective mechanism may depend on the state of metabolism, cell proliferation, and other traits that distinguish a normal cell from a cancerous one. Indeed, TACR1 inhibitors were discussed as possible anticancer agents. ${ }^{75}$ Second, it has been argued that death signaling is commonly impaired in malignancies, so protection of normal cells through targeting of these same pathways may not affect the efficacy of 
treatment, at least, for some cancers. ${ }^{76}$ Third, an acute nephroprotective intervention may be too short to sustain tumor growth. In fact, there are examples of experimental drugs that protect normal cells from temporary stresses by inhibiting tumor suppressors without long-term oncogenic consequences. ${ }^{77}$ Similar approaches for nephroprotection have yielded promising early results. ${ }^{78}$

The differential effects on cisplatin resistance, as well as the differences in the ability to enhance the effect of NK1R inhibitors argue that the identified genes affect multiple pathways of cell death. In addition to achieving maximal protection by a combination therapy, an ability to influence the outcome through several distinct mechanisms provides more options to balance benefits and risks for a given patient. These considerations support further investigation of multiple pathways, elements of which have been uncovered in the current study.

\section{Materials and Methods}

Cell culture and viral transduction. HK-2 cells ${ }^{19}$ were grown in keratinocyte serum-free medium (Life Technologies, Grand Island, NY, USA), supplemented with 10\% fetal bovine serum (Atlanta Biologicals, Norcross, GA, USA), $5 \mathrm{ng} / \mathrm{ml}$ epidermal growth factor (Life Technologies), and $100 \mathrm{U} / \mathrm{ml}$ penicillin/ streptomycin (Life Technologies). HKC-8 ${ }^{21}$ were maintained in Dulbecco's modified Eagle's medium (Life Technologies) supplemented with $10 \%$ fetal bovine serum and $100 \mathrm{U} / \mathrm{ml}$ penicillin/streptomycin. Viral transduction was performed as described. ${ }^{79}$

In vitro hypoxia treatment. The cells were subjected to low-glucose medium (Life Technologies) and $0.2 \%$ hypoxia for $48 \mathrm{~h}$ in a Biospherix Xvivo system (Lacona, NY, USA). Following treatment, the cells were fixed, their numbers were assessed using methylene blue staining and the extraction method, ${ }^{80}$ and the values were calculated as percentage of cell number in parallel normoxic cultures. The values for the cultures transduced with various constructs were normalized for the values corresponding to the parental cells. The individual shRNA sequences and their relative effects on HK-2 cells are shown in Supplementary Figure 2.

Cisplatin treatment. Kidney epithelial cells expressing candidate shRNAs and the respective controls were exposed to cisplatin (Sigma-Aldrich, St. Louis, MO, USA) at various concentrations $(0,0.1,0.2,0.4,0.8,1.6,3.2,6.4,12.8$, and $25.6 \mu \mathrm{M}$ ) in triplicates for $48 \mathrm{~h}$. Subsequently, the cells were washed and cultured in fresh medium for an additional 3 days. At that time, the cells were fixed, and their numbers were assessed using methylene blue staining and the extraction method. ${ }^{80}$ The values were calculated as percentage of untreated cell number, and the $I_{50}$ values were computed using GraphPad Prizm 6 (GraphPad Software, Inc., La Jolla, CA, USA).

In vitro treatment with NK1R antagonists. The cells were treated with various concentrations of L-733,060 (Tocris, Bristol, UK) and Aprepitant (Selleck Chemicals, Houston, TX, USA) for $1 \mathrm{~h}$ before being subjected to hypoxia treatment as described above.

Quantitative RT-PCR. The transcripts were detected using quantitative realtime RT-PCR using GAPDH as an endogenous control. The nucleotide sequences were: RHOB 5'-ACATTGAGGTGGACGGCAAGCA-3' and 5'-CTGTCCACCGAGAA GCACATGA-3'; BCL2L14 5'-GTAACTGAGGGTCTCTCCTTCC-3' and 5'-GGAATG GGGATGAAGGCAGTGT-3'; SCIN 5'-GCAGAGTATGTAGCAAGTGTCCT-3' and 5'-GTAAAGCCGAGGTGGATGGTCT-3'; TACR1 5'-GCCTGTTCTACTGGAAGTC CAC-3 and 5'-CACAGATGACCACTTTGGTGGC-3'; GCNT35'-CACCAGAGACTGT GAGCACTTC-3' and 5'-CATACACAGCTCGCAGTAGCCT-3' ECT2 5'-GCAGTCAG CAAGGTGGCAAGTT-3' and 5'-CTCTGGTGCAAGGATAGGTCCA-3'; GAPDH 5'-GTCTCCTCTGACTTCAACGCG-3' and 5'ACCACCCTGTTGCTGTAGCAA. RNA was isolated using the RNeasy, RT kit (Qiagen, Germantown, MD, USA). Complementary DNA was synthesized using SuperScript III (Life Technologies). PCRs were performed using an $A B I$ Prism 7900 Sequence Detection System (Waltham, MA, USA) and IQ SYBR Green SuperMix (Bio-Rad, Hercules, CA, USA). The thermal cycling conditions comprised $2 \mathrm{~min}$ at $50^{\circ} \mathrm{C}, 10 \mathrm{~min}$ at $90^{\circ} \mathrm{C}$, and $1 \mathrm{~min}$ at $60^{\circ} \mathrm{C}$ for 40 cycles. Data were analyzed using $\mathrm{RQ}$ Manager 1.2.1 (ABI).
In vivo model of ischemia. The work was approved by the Committee for Animal Experiments of Basel (TierversuchsKommission von BaselStadt). C57BL/6 mice were pretreated with various doses of TACR1 inhibitor or the corresponding vehicle. After $30 \mathrm{~min}$, mice were anesthetized (with ketamine/rompun). Both kidneys were exposed through flank incisions and kidney pedicles were clamped with atraumatic clamps while the mice were kept at $36^{\circ} \mathrm{C}$. The clamps were removed after $25 \mathrm{~min}$, and the wounds were closed in two layers. After $24 \mathrm{~h}$, blood samples were assayed for the markers of kidney injury.

Caspase activity assay. Apo-ONE Homogeneous Caspase- $3 / 7$ Assay (Promega, Madison, WI, USA) was applied to HK-2 cell lysates as per the manufacturer's protocol.

\section{Conflict of Interest}

BS, SG, EW, GDN and ME are employees of F. Hoffmann-La Roche AG. The remaining authors declare no conflict of interest.

Acknowledgements. The study was supported in part by the Roswell Park Alliance Foundation. We thank $\operatorname{Dr} A$ Chenchik and $\operatorname{Dr} M$ Makhanov for their assistance in analyzing the results of the shRNA screening, and DrW Burhans for his help in preparing the manuscript. This study was supported in part by funds from F. Hoffmann-La Roche AG.

1. Devarajan P. Update on mechanisms of ischemic acute kidney injury. J Am Soc Nephrol 2006; 17: 1503-1520.

2. Lameire NH, Bagga A, Cruz D, De Maeseneer J, Endre Z, Kellum JA et al. Acute kidney injury: an increasing global concern. Lancet 2013; 382: 170-179.

3. Singbartl K, Kellum JA. AKI in the ICU: definition, epidemiology, risk stratification, and outcomes. Kidney Int 2012; 81: 819-825.

4. Munshi R, Hsu C, Himmelfarb J. Advances in understanding ischemic acute kidney injury. BMC Med 2011; 9: 11.

5. Evans RG, Goddard D, Eppel GA, O'Connor PM. Factors that render the kidney susceptible to tissue hypoxia in hypoxemia. Am J Physiol Regul Integr Comp Physiol 2011; 300: R931-R940.

6. Haase VH. Regulation of erythropoiesis by hypoxia-inducible factors. Blood Rev 2013; 27: 41-53.

7. Bonventre JV, Yang L. Cellular pathophysiology of ischemic acute kidney injury. J Clin Invest 2011; 121: 4210-4221.

8. Palevsky PM. Chronic-on-acute kidney injury. Kidney Int 2012; 81: 430-431.

9. Demirjian S, Schold JD, Navia J, Mastracci TM, Paganini EP, Yared JP et al. Predictive models for acute kidney injury following cardiac surgery. Am J Kidney Dis 2012; 59: 382-389.

10. Dirkes S. Sepsis and inflammation: impact on acute kidney injury. Nephrol Nurs J 2013; 40: 125-132; quiz 133.

11. Porter GA. Risk factors for toxic nephropathies. Toxicol Lett 1989; 46: 269-279.

12. Siedlecki A, Irish W, Brennan DC. Delayed graft function in the kidney transplant. Am J Transplant 2011; 11: 2279-2296.

13. Kusch A, Hoff U, Bubalo G, Zhu Y, Fechner M, Schmidt-Ullrich $R$ et al. Novel signalling mechanisms and targets in renal ischaemia and reperfusion injury. Acta Physiol (Oxf) 2013; 208: $25-40$.

14. Remuzzi G, Benigni A, Finkelstein FO, Grunfeld JP, Joly D, Katz I et al. Kidney failure: aims for the next 10 years and barriers to success. Lancet 2013; 382: 353-362.

15. Sharfuddin AA, Molitoris BA. Pathophysiology of ischemic acute kidney injury. Nat Rev Nephrol 2011; 7: 189-200.

16. Lieberthal W, Nigam SK. Acute renal failure. I. Relative importance of proximal vs. distal tubular injury. Am J Physiol 1998; 275: F623-F631.

17. Hammond EM, Giaccia AJ. The role of p53 in hypoxia-induced apoptosis. Biochem Biophys Res Commun 2005; 331: 718-725.

18. Gartel AL, Kandel ES. RNA interference in cancer. Biomol Eng 2006; 23: 17-34.

19. Ryan MJ, Johnson G, Kirk J, Fuerstenberg SM, Zager RA, Torok-Storb B. HK-2: an immortalized proximal tubule epithelial cell line from normal adult human kidney. Kidney Int 1994; 45: 48-57.

20. Fujiki K, Inamura H, Matsuoka M. Detrimental effects of Notch1 signaling activated by cadmium in renal proximal tubular epithelial cells. Cell Death Dis 2014; 5: e1378.

21. Racusen LC, Monteil C, Sgrignoli A, Lucskay M, Marouillat S, Rhim JG et al. Cell lines with extended in vitro growth potential from human renal proximal tubule: characterization, response to inducers, and comparison with established cell lines. J Lab Clin Med 1997; 129: 318-329.

22. Cost NG, Adibi M, Lubahn JD, Romman A, Raj GV, Sagalowsky Al et al. Effect of testicular germ cell tumor therapy on renal function. Urology 2012; 80: 641-648.

23. Beroukhim R, Brunet JP, Di Napoli A, Mertz KD, Seeley A, Pires MM et al. Patterns of gene expression and copy-number alterations in von-hippel lindau disease-associated and sporadic clear cell carcinoma of the kidney. Cancer Res 2009; 69: 4674-4681.

24. Gumz ML, Zou H, Kreinest PA, Childs AC, Belmonte LS, LeGrand SN et al. Secreted frizzledrelated protein 1 loss contributes to tumor phenotype of clear cell renal cell carcinoma. Clin Cancer Res 2007; 13: 4740-4749. 
25. Padanilam BJ. Cell death induced by acute renal injury: a perspective on the contributions of apoptosis and necrosis. Am J Physiol Renal Physiol 2003; 284: F608-F627.

26. Carmeliet P, Dor Y, Herbert JM, Fukumura D, Brusselmans K, Dewerchin M et al. Role of HIF-1alpha in hypoxia-mediated apoptosis, cell proliferation and tumour angiogenesis. Nature 1998; 394: 485-490.

27. Malhotra R, Tyson DW, Rosevear HM, Brosius FC 3rd. Hypoxia-inducible factor-1alpha is a critical mediator of hypoxia induced apoptosis in cardiac $\mathrm{H} 9 \mathrm{c} 2$ and kidney epithelial HK-2 cells. BMC Cardiovasc Disord 2008; 8: 9 .

28. Portilla D. Carnitine palmitoyl-transferase enzyme inhibition protects proximal tubules during hypoxia. Kidney Int 1997; 52: 429-437.

29. Persy VP, Verhulst A, Ysebaert DK, De Greef KE, De Broe ME. Reduced postischemic macrophage infiltration and interstitial fibrosis in osteopontin knockout mice. Kidney Int 2003; 63: $543-553$

30. Pallet N, Rabant M, Legendre C, Martinez F, Choukroun $G$. The nephroprotective properties of recombinant human erythropoietin in kidney transplantation: experimental facts and clinical proofs. Am J Transplant 2012; 12: 3184-3190.

31. Saenz-Morales D, Conde E, Blanco-Sanchez I, Ponte B, Aguado-Fraile E, de Las Casas G et al. Differential resolution of inflammation and recovery after renal ischemia-reperfusion injury in Brown Norway compared with Spraque Dawley rats. Kidney Int 2010; 77: 781-793.

32. Negi S, Shigematsu T. Current therapeutic strategies for acute kidney injury. Clin Exp Nephrol 2012; 16: 672-678.

33. Kaelin WG Jr. Molecular biology. Use and abuse of RNAi to study mammalian gene function. Science 2012; 337: 421-422.

34. Badiola N, Malagelada C, Llecha N, Hidalgo J, Comella JX, Sabria J et al. Activation of caspase- 8 by tumour necrosis factor receptor 1 is necessary for caspase- 3 activation and apoptosis in oxygen-glucose deprived cultured cortical cells. Neurobiol Dis 2009; 35: 438-447.

35. Chiao $\mathrm{H}$, Caldwell RW. The role of substance $P$ in myocardial dysfunction during ischemia and reperfusion. Naunyn Schmiedeberg's Arch Pharmacoly 1996; 353: 400-407.

36. Turner RJ, Helps SC, Thornton E, Vink R. A substance P antagonist improves outcome when administered $4 \mathrm{~h}$ after onset of ischaemic stroke. Brain Res 2011; 1393: 84-90.

37. Castro-Obregon S, Del Rio G, Chen SF, Swanson RA, Frankowski H, Rao RV et al. A ligandreceptor pair that triggers a non-apoptotic form of programmed cell death. Cell Death Differ 2002; 9: 807-817.

38. Vandenabeele $P$, Melino $G$. The flick of a switch: which death program to choose? Cell Death Differ 2012; 19: 1093-1095.

39. Park TJ, Comer C, Carol A, Lu Y, Hong HS, Rice FL. Somatosensory organization and behavior in naked mole-rats: II. Peripheral structures, innervation, and selective lack of neuropeptides associated with thermoregulation and pain. J Comp Neurol 2003; 465: 104-120.

40. Stone EL, Lee SH, Ismail MN, Fukuda M. Characterization of mice with targeted deletion of the gene encoding core 2 beta1,6- N-acetylglucosaminyltransferase-2. Methods Enzymol 2010; 479: 155-172.

41. Barbetti V, Tusa I, Cipolleschi MG, Rovida E, Dello Sbarba P. AML1/ETO sensitizes via TRAIL acute myeloid leukemia cells to the pro-apoptotic effects of hypoxia. Cell Death Dis 2013; 4: e536.

42. Adachi T, Sugiyama N, Gondai T, Yagita H, Yokoyama T. Blockade of death ligand TRAIL inhibits renal ischemia reperfusion injury. Acta Histochem Cytochem 2013; 46: 161-170.

43. Wagner KW, Punnoose EA, Januario T, Lawrence DA, Pitti RM, Lancaster $\mathrm{K}$ et al. Deathreceptor O-glycosylation controls tumor-cell sensitivity to the proapoptotic ligand Apo2L/ TRAIL. Nat Med 2007; 13: 1070-1077.

44. Lichtenstein RG, Rabinovich GA. Glycobiology of cell death: when glycans and lectins govern cell fate. Cell Death Differ 2013; 20: 976-986.

45. Meshki J, Douglas SD, Hu M, Leeman SE, Tuluc F. Substance $P$ induces rapid and transient membrane blebbing in U373MG cells in a p21-activated kinase-dependent manner. PLoS One 2011; 6: e25332.

46. Somanath PR, Vijai J, Kichina JV, Byzova T, Kandel ES. The role of PAK-1 in activation of MAP kinase cascade and oncogenic transformation by Akt. Oncogene 2009; 28: 2365-2369.

47. Drosopoulos KG, Roberts ML, Cermak L, Sasazuki T, Shirasawa S, Andera L et Transformation by oncogenic RAS sensitizes human colon cells to TRAIL-induced apoptosis by up-regulating death receptor 4 and death receptor 5 through a MEK-dependent pathway. J Biol Chem 2005; 280: 22856-22867.

48. Dong C, Yuan T, Wu Y, Wang Y, Fan TW, Miriyala S et al. Loss of FBP1 by Snail-mediated repression provides metabolic advantages in basal-like breast cancer. Cancer Cell 2013; 23: 316-331.

49. Li B, Qiu B, Lee DS, Walton ZE, Ochocki JD, Mathew LK et al. Fructose-1,6-bisphosphatase opposes renal carcinoma progression. Nature 2014; 513: 251-255.

50. Grynberg A. Effectors of fatty acid oxidation reduction: promising new anti-ischaemic agents. Curr Pharm Des 2005; 11: 489-509.

51. Ceccarelli SM, Chomienne O, Gubler M, Arduini A. Carnitine palmitoyltransferase (CPT) modulators: a medicinal chemistry perspective on 35 years of research. J Med Chem 2011; 54: 3109-3152.

52. Gdynia G, Lehmann-Koch J, Sieber S, Tagscherer KE, Fassl A, Zentgraf $\mathrm{H}$ et al. BLOC1S2 interacts with the HIPPI protein and sensitizes NCH89 glioblastoma cells to apoptosis. Apoptosis 2008; 13: 437-447.
53. Lejen T, Pene TD, Rose SD, Trifaro JM. The role of different Scinderin domains in the control of F-actin cytoskeleton during exocytosis. Ann N Y Acad Sci 2002; 971 248-250.

54. Zunino R, Li Q, Rose SD, Romero-Benitez MM, Lejen T, Brandan NC et al. Expression of scinderin in megakaryoblastic leukemia cells induces differentiation, maturation, and apoptosis with release of plateletlike particles and inhibits proliferation and tumorigenesis. Blood 2001; 98: 2210-2219.

55. Milner CM, Higman VA, Day AJ. TSG-6: a pluripotent inflammatory mediator? Biochem Soc Trans 2006; 34: 446-450.

56. Gao G, Zhang B, Ramesh G, Betterly D, Tadagavadi RK, Wang W et al. TNF-alpha mediates increased susceptibility to ischemic AKI in diabetes. Am J Physiol Renal Physiol 2013; 304: F515-F521.

57. Lesley J, Gal I, Mahoney DJ, Cordell MR, Rugg MS, Hyman R et al. TSG-6 modulates the interaction between hyaluronan and cell surface CD44. J Biol Chem 2004; 279 : 25745-25754.

58. Colombaro V, Decleves AE, Jadot I, Voisin V, Giordano L, Habsch I et al. Inhibition of hyaluronan is protective against renal ischaemia-reperfusion injury. Nephrol Dial Transplant 2013; 28: 2484-2493

59. Rouschop KM, Roelofs JJ, Claessen N, da Costa Martins P, Zwaginga JJ, Pals ST et al. Protection against renal ischemia reperfusion injury by CD44 disruption. J Am Soc Nephrol 2005; 16: 2034-2043.

60. Bommaya G, Meran S, Krupa A, Phillips AO, Steadman R. Tumour necrosis factorstimulated gene (TSG)- 6 controls epithelial-mesenchymal transition of proximal tubular epithelial cells. Int J Biochem Cell Biol 2011; 43: 1739-1746.

61. Cimbora-Zovko T, Fritz G, Mikac N, Osmak M. Downregulation of RhoB GTPase confers resistance to cisplatin in human laryngeal carcinoma cells. Cancer Lett 2010; 295: 182-190.

62. Huang M, Prendergast GC. RhoB in cancer suppression. Histol Histopathol 2006; 21 213-218.

63. Skuli N, Monferran S, Delmas C, Lajoie-Mazenc I, Favre G, Toulas C et al. Activation of RhoB by hypoxia controls hypoxia-inducible factor-1alpha stabilization through glycogen synthase kinase-3 in U87 glioblastoma cells. Cancer Res 2006; 66: 482-489.

64. Avivi A, Brodsky L, Nevo E, Band MR. Differential expression profiling of the blind subterranean mole rat Spalax ehrenbergi superspecies: bioprospecting for hypoxia tolerance. Physiol Genomics 2006; 27: 54-64.

65. Mazurek N, Byrd JC, Sun Y, Hafley M, Ramirez K, Burks J et al. Cell-surface galectin-3 confers resistance to TRAIL by impeding trafficking of death receptors in metastatic colon adenocarcinoma cells. Cell Death Differ 2012; 19: 523-533.

66. Jin Z, McDonald ER 3rd, Dicker DT, El-Deiry WS. Deficient tumor necrosis factor-related apoptosis-inducing ligand (TRAIL) death receptor transport to the cell surface in human colon cancer cells selected for resistance to TRAIL-induced apoptosis. J Biol Chem 2004 279: 35829-35839.

67. Guo B, Godzik A, Reed JC. Bcl-G, a novel pro-apoptotic member of the Bcl-2 family. J Biol Chem 2001; 276: 2780-2785.

68. Giam M, Okamoto T, Mintern JD, Strasser A, Bouillet P. Bcl-2 family member Bcl-G is not a proapoptotic protein. Cell Death Dis 2012; 3: e404.

69. Tischner D, Villunger A. Bcl-G acquitted of murder!. Cell Death Dis 2012; 3: e405.

70. Fang X, Seim I, Huang Z, Gerashchenko MV, Xiong Z, Turanov AA et al. Adaptations to a subterranean environment and longevity revealed by the analysis of mole rat genomes. Cell Rep 2014; 8: 1354-1364.

71. Woods NT, Mesquita RD, Sweet M, Carvalho MA, Li X, Liu Y et al. Charting the landscape of tandem BRCT domain-mediated protein interactions. Sci Signal 2012; 5: rs6.

72. Arany I, Safirstein RL. Cisplatin nephrotoxicity. Semin Nephrol 2003; 23: 460-464.

73. Pickard MR, Mourtada-Maarabouni M, Williams GT. Candidate tumour suppressor Fau regulates apoptosis in human cells: an essential role for Bcl-G. Biochim Biophys Acta 2011; 1812: $1146-1153$.

74. Srougi MC, Burridge K. The nuclear guanine nucleotide exchange factors Ect2 and Net1 regulate RhoB-mediated cell death after DNA damage. PLoS One 2011; 6: e17108.

75. Munoz M, Rosso M, Covenas R, Montero I, Gonzalez-Moles MA, Robles MJ. Neurokinin-1 receptors located in human retinoblastoma cell lines: antitumor action of its antagonist, L-732,138. Invest Ophthalmol Vis Sci 2007; 48: 2775-2781.

76. Komarova EA, Gudkov AV. Chemoprotection from p53-dependent apoptosis: potential clinical applications of the p53 inhibitors. Biochem Pharmacol 2001; 62: 657-667.

77. Komarov PG, Komarova EA, Kondratov RV, Christov-Tselkov K, Coon JS, Chernov MV et al. A chemical inhibitor of $p 53$ that protects mice from the side effects of cancer therapy. Science 1999; 285: 1733-1737.

78. Molitoris BA, Dagher PC, Sandoval RM, Campos SB, Ashush H, Fridman E et al. siRNA targeted to $\mathrm{p53}$ attenuates ischemic and cisplatin-induced acute kidney injury. J Am Soc Nephrol 2009; 20: 1754-1764.

79. Singhal R, Deng $X$, Chenchik AA, Kandel ES. Long-distance effects of insertional mutagenesis. PLOS One 2011; 6: e15832.

80. Singhal R, Kandel ES. The response to PAK1 inhibitor IPA3 distinguishes between cancer cells with mutations in BRAF and Ras oncogenes. Oncotarget 2012; 3: 700-708. 